\title{
Some results for Apostol-type polynomials associated with umbral algebra
}

\author{
Da-Qian Lu' ${ }^{1}$ Chang-He Xiang ${ }^{2}$ and Qiu-Ming Luo ${ }^{2 *}$
}

\section{"Correspondence:}

luomath2007@163.com

${ }^{2}$ Department of Mathematics, Chongqing Normal University,

Chongqing Higher Education Mega Center, Huxi Campus, Chongqing,

401331, People's Republic of China

Full list of author information is

available at the end of the article

\begin{abstract}
A family of the Apostol-type polynomials was introduced and investigated recently by Luo and Srivastava (see (Appl. Math. Comput. 217:5702-5728, 2011)). In this paper, we study this polynomial family on $P$, the algebra of polynomials in a single variable $x$ over all linear functional on $P$. By using the way of the umbral algebra, we obtain some fundamental properties of the generalized Apostol-type polynomials. We also show some special cases which include the corresponding results of Dere and Simsek etc. MSC: Primary 05A40; secondary 11B68; 05A10; 05A15
\end{abstract}

Keywords: generalized Apostol-type polynomials; Sheffer sequences and Appell sequences; umbral algebra; Stirling numbers

\section{Introduction, definitions and motivation}

Throughout this paper, we make use of the following conventional notations: $\mathbb{N}=$ $\{1,2,3, \ldots\}$ denotes the set of natural numbers, $\mathbb{C}$ denotes the set of complex numbers.

The classical Bernoulli polynomials $B_{n}(x)$, the classical Euler polynomials $E_{n}(x)$ and the classical Genocchi polynomials $G_{n}(x)$, together with their familiar generalizations $B_{n}^{(\alpha)}(x)$, $E_{n}^{(\alpha)}(x)$ and $G_{n}^{(\alpha)}(x)$ of order $\alpha$, are usually defined by means of the following generating functions (see, for details, [1, pp.532-533] and [2]):

$$
\begin{aligned}
& \left(\frac{z}{e^{z}-1}\right)^{\alpha} e^{x z}=\sum_{n=0}^{\infty} B_{n}^{(\alpha)}(x) \frac{z^{n}}{n !} \quad(|z|<2 \pi), \\
& \left(\frac{2}{e^{z}+1}\right)^{\alpha} e^{x z}=\sum_{n=0}^{\infty} E_{n}^{(\alpha)}(x) \frac{z^{n}}{n !} \quad(|z|<\pi)
\end{aligned}
$$

and

$$
\left(\frac{2 z}{e^{z}+1}\right)^{\alpha} e^{x z}=\sum_{n=0}^{\infty} G_{n}^{(\alpha)}(x) \frac{z^{n}}{n !} \quad(|z|<\pi) .
$$

It is easy to see that $B_{n}(x), E_{n}(x)$ and $G_{n}(x)$ are given, respectively, by

$$
\begin{aligned}
& B_{n}(x):=B_{n}^{(1)}(x), \quad E_{n}(x):=E_{n}^{(1)}(x) \quad \text { and } \\
& G_{n}(x):=G_{n}^{(1)}(x) \quad\left(n \in \mathbb{N}_{0}:=\mathbb{N} \cup\{0\}\right) .
\end{aligned}
$$

C 2013 Lu et al.; licensee Springer. This is an Open Access article distributed under the terms of the Creative Commons Attribution License (http://creativecommons.org/licenses/by/2.0), which permits unrestricted use, distribution, and reproduction in any medium, provided the original work is properly cited. 
For the classical Bernoulli numbers $B_{n}$, the classical Euler numbers $E_{n}$ and the classical Genocchi numbers $G_{n}$ of order $n$, we have

$$
B_{n}:=B_{n}(0)=B_{n}^{(1)}(0), \quad E_{n}:=E_{n}(0)=E_{n}^{(1)}(0) \quad \text { and } \quad G_{n}:=G_{n}(0)=G_{n}^{(1)}(0),
$$

respectively.

Some interesting analogues of the classical Bernoulli polynomials and numbers were first investigated by Apostol (see [3, p.165, Eq. (3.1)]) and (more recently) by Srivastava (see [4, pp.83-84]). We begin by recalling Apostol's definitions as follows.

Definition 1.1 (Apostol [3]; see also Srivastava [4]) The Apostol-Bernoulli polynomials $\mathcal{B}_{n}(x ; \lambda)(\lambda \in \mathbb{C})$ are defined by means of the following generating function:

$$
\begin{aligned}
& \frac{z e^{x z}}{\lambda e^{z}-1}=\sum_{n=0}^{\infty} \mathcal{B}_{n}(x ; \lambda) \frac{z^{n}}{n !} \\
& \quad(|z|<2 \pi \text { when } \lambda=1 ;|z|<|\log \lambda| \text { when } \lambda \neq 1)
\end{aligned}
$$

with, of course,

$$
B_{n}(x)=\mathcal{B}_{n}(x ; 1) \quad \text { and } \quad \mathcal{B}_{n}(\lambda):=\mathcal{B}_{n}(0 ; \lambda)
$$

where $\mathcal{B}_{n}(\lambda)$ denotes the so-called Apostol-Bernoulli numbers.

Recently, Luo and Srivastava [5] further extended the Apostol-Bernoulli polynomials as the so-called Apostol-Bernoulli polynomials of order $\alpha$.

Definition 1.2 (Luo and Srivastava [5]) The Apostol-Bernoulli polynomials $\mathcal{B}_{n}^{(\alpha)}(x ; \lambda)(\lambda \in$ $\mathbb{C})$ of order $\alpha(\alpha \in \mathbb{N})$ are defined by means of the following generating function:

$$
\begin{aligned}
& \left(\frac{z}{\lambda e^{z}-1}\right)^{\alpha} \cdot e^{x z}=\sum_{n=0}^{\infty} \mathcal{B}_{n}^{(\alpha)}(x ; \lambda) \frac{z^{n}}{n !} \\
& \quad(|z|<2 \pi \text { when } \lambda=1 ;|z|<|\log \lambda| \text { when } \lambda \neq 1)
\end{aligned}
$$

with, of course,

$$
B_{n}^{(\alpha)}(x)=\mathcal{B}_{n}^{(\alpha)}(x ; 1) \quad \text { and } \quad \mathcal{B}_{n}^{(\alpha)}(\lambda):=\mathcal{B}_{n}^{(\alpha)}(0 ; \lambda)
$$

where $\mathcal{B}_{n}^{(\alpha)}(\lambda)$ denotes the so-called Apostol-Bernoulli numbers of order $\alpha$.

In this sequel, Luo [6] gave an analogous extension of the generalized Euler polynomials which is the so-called Apostol-Euler polynomials of order $\alpha$.

Definition 1.3 (Luo [6]) The Apostol-Euler polynomials $\mathcal{E}_{n}^{(\alpha)}(x ; \lambda)$ of order $\alpha(\alpha, \lambda \in \mathbb{C})$ are defined by means of the following generating function:

$$
\left(\frac{2}{\lambda e^{z}+1}\right)^{\alpha} \cdot e^{x z}=\sum_{n=0}^{\infty} \mathcal{E}_{n}^{(\alpha)}(x ; \lambda) \frac{z^{n}}{n !} \quad(|z|<|\log (-\lambda)|)
$$


with, of course,

$$
E_{n}^{(\alpha)}(x)=\mathcal{E}_{n}^{(\alpha)}(x ; 1) \quad \text { and } \quad \mathcal{E}_{n}^{(\alpha)}(\lambda):=\mathcal{E}_{n}^{(\alpha)}(0 ; \lambda)
$$

where $\mathcal{E}_{n}^{(\alpha)}(\lambda)$ denotes the so-called Apostol-Euler numbers of order $\alpha$.

On the subject of the Genocchi polynomials $G_{n}(x)$ and their various extensions, a remarkably large number of investigations have appeared in the literature (see, for example, [7-11]). Moreover, Luo (see [12]) introduced and investigated the Apostol-Genocchi polynomials of (real or complex) order $\alpha$, which are defined as follows.

Definition 1.4 The Apostol-Genocchi polynomials $\mathcal{G}_{n}^{(\alpha)}(x ; \lambda)(\lambda \in \mathbb{C})$ of order $\alpha(\alpha \in \mathbb{N})$ are defined by means of the following generating function:

$$
\left(\frac{2 z}{\lambda e^{z}+1}\right)^{\alpha} \cdot e^{x z}=\sum_{n=0}^{\infty} \mathcal{G}_{n}^{(\alpha)}(x ; \lambda) \frac{z^{n}}{n !} \quad(|z|<|\log (-\lambda)|)
$$

with, of course,

$$
\begin{aligned}
& G_{n}^{(\alpha)}(x)=\mathcal{G}_{n}^{(\alpha)}(x ; 1), \quad \mathcal{G}_{n}^{(\alpha)}(\lambda):=\mathcal{G}_{n}^{(\alpha)}(0 ; \lambda), \\
& \mathcal{G}_{n}(x ; \lambda):=\mathcal{G}_{n}^{(1)}(x ; \lambda) \quad \text { and } \quad \mathcal{G}_{n}(\lambda):=\mathcal{G}_{n}^{(1)}(\lambda),
\end{aligned}
$$

where $\mathcal{G}_{n}(\lambda), \mathcal{G}_{n}^{(\alpha)}(\lambda)$ and $\mathcal{G}_{n}(x ; \lambda)$ denote the so-called Apostol-Genocchi numbers, the Apostol-Genocchi numbers of order $\alpha$ and the Apostol-Genocchi polynomials, respectively.

Ozden et al. [13] introduced and investigated the following unification (and generalization) of the generating functions of the three families of Apostol-type polynomials:

$$
\begin{aligned}
& \frac{2^{1-\kappa} z^{\kappa}}{\beta^{b} e^{z}-a^{b}} e^{x z}=\sum_{n=0}^{\infty} \mathcal{Y}_{n, \beta}(x ; \kappa, a, b) \frac{z^{n}}{n !} \\
& \quad(|z|<2 \pi \text { when } \beta=a ;|z|<|b \log (\beta / a)| \text { when } \beta \neq a ; \kappa, \beta \in \mathbb{C} ; a, b \in \mathbb{C} \backslash\{0\}) .
\end{aligned}
$$

It is found from [14] that Ozden further gave an extension of the above definition (1.14) as follows:

\section{Definition 1.5}

$$
\begin{aligned}
& \left(\frac{2^{1-\kappa} z^{\kappa}}{\beta^{b} e^{z}-a^{b}}\right)^{\alpha} e^{x z}=\sum_{n=0}^{\infty} \mathcal{Y}_{n, \beta}^{(\alpha)}(x ; \kappa, a, b) \frac{z^{n}}{n !} \\
& (\alpha \in \mathbb{N} ;|z|<2 \pi \text { when } \beta=a ;|z|<|b \log (\beta / a)| \text { when } \beta \neq a ; \\
& \kappa, \beta \in \mathbb{C} ; a, b \in \mathbb{C} \backslash\{0\}) .
\end{aligned}
$$

The author [15] obtained a unified relation between the $\mathcal{Y}_{n, \beta}^{(\alpha)}(x ; \kappa, a, b)$ and the Gauss hypergeometric function ${ }_{2} F_{1}(a, b ; c ; z)$, and gave some identities of $\mathcal{Y}_{n, \beta}^{(\alpha)}(x ; \kappa, a, b)$. 
Recently, Luo and Srivastava [16] introduced more general unification (and generalization) of the above-mentioned three families of the generalized Apostol-type polynomials.

Definition 1.6 (Luo and Srivastava [16]) The generalized Apostol-type polynomials $\mathcal{F}_{n}^{(\alpha)}(x ; \lambda ; \mu ; \nu)(\alpha \in \mathbb{N} ; \lambda, \mu, v \in \mathbb{C})$ of order $\alpha$ are defined by means of the following generating function:

$$
\left(\frac{2^{\mu} z^{\nu}}{\lambda e^{z}+1}\right)^{\alpha} e^{x z}=\sum_{n=0}^{\infty} \mathcal{F}_{n}^{(\alpha)}(x ; \lambda ; \mu ; \nu) \frac{z^{n}}{n !} \quad(|z|<|\log (-\lambda)|) .
$$

Clearly, we have

$$
\begin{aligned}
& \mathcal{B}_{n}^{(\alpha)}(x ; \lambda)=(-1)^{\alpha} \mathcal{F}_{n}^{(\alpha)}(x ;-\lambda ; 0 ; 1) \quad(\alpha \in \mathbb{N}), \\
& \mathcal{E}_{n}^{(\alpha)}(x ; \lambda)=\mathcal{F}_{n}^{(\alpha)}(x ; \lambda ; 1 ; 0) \quad(\alpha \in \mathbb{C}), \\
& \mathcal{G}_{n}^{(\alpha)}(x ; \lambda)=\mathcal{F}_{n}^{(\alpha)}(x ; \lambda ; 1 ; 1) \quad(\alpha \in \mathbb{N}), \\
& \mathcal{Y}_{n, \beta}(x ; \kappa, a, b)=-\frac{1}{a^{b}} \mathcal{F}_{n}^{(1)}\left(x ;-\left(\frac{\beta}{a}\right)^{b} ; 1-\kappa ; \kappa\right)
\end{aligned}
$$

and

$$
\mathcal{Y}_{n, \beta}^{(\alpha)}(x ; \kappa, a, b)=(-1)^{\alpha} \frac{1}{a^{b \alpha}} \mathcal{F}_{n}^{(\alpha)}\left(x ;-\left(\frac{\beta}{a}\right)^{b} ; 1-\kappa ; \kappa\right) .
$$

In $[5,6,17,18]$, the authors have researched some elementary properties of the Apostoltype polynomials, and some relationships among the Apostol-type polynomials. More investigations about this subject can be found in $[13,15,16,19-30]$.

The aim of this paper is to study the generalized Apostol-type polynomials $\mathcal{F}_{n}^{(\alpha)}(x ; \lambda ; \mu ; v)$ on the umbral algebra by using the way as the reference [31-33]. We research some fundamental properties of this polynomial family. Some special cases, which include the corresponding results [31-33], are also considered.

\section{Umbral algebra of Roman}

We can use the following notations and definitions, which are given by Roman [34, pp.1-125].

Let $P$ be the algebra of polynomials in a single variable $x$ over the field of complex numbers. Let $P^{*}$ be the vector space of all linear functionals on $P$. Let $\langle L \mid p(x)\rangle$ be the action of a linear functional $L$ on a polynomial $p(x)$. Let $\mathcal{F}$ denote the algebra of formal power series

$$
f(t)=\sum_{k=0}^{\infty} \frac{a_{k}}{k !} t^{k}
$$

Such algebra is called umbral algebra. Each $f \in \mathcal{F}$ defines a linear functional on $P$ and

$$
a_{k}=\left\langle f(t) \mid t^{k}\right\rangle
$$

for all $k \geq 0$. 
The order $o(f(t))$ of a power series $f(t)$ is the smallest integer $k$ for which the coefficient of $t^{k}$ does not vanish. A series $f(t)$ for which $o(f(t))=1$ will be called a delta series. When we are considering a delta series $f(t)$ in $\mathcal{F}$ as a linear functional, we will refer to it as a delta functional.

It is well known that $\left\langle t^{k} \mid x^{n}\right\rangle=n ! \delta_{n, k}$, where $\delta_{n, k}$ denotes the Kronecker symbol. For all $f(t)$ in $\mathcal{F}$,

$$
f(t)=\sum_{k=0}^{\infty} \frac{\left\langle f(t) \mid x^{k}\right\rangle}{k !} t^{k} .
$$

Let $f(t)$ and $g(t)$ be in $\mathcal{F}$. Then we have

$$
\langle f(t) g(t) \mid p(x)\rangle=\langle f(t) \mid g(t) p(x)\rangle
$$

For $y \in \mathbb{C}$, then the evaluation functional is defined to be the power series $e^{y t}$. By (2.2), we have

$$
\left\langle e^{y t} \mid p(x)\right\rangle=p(y)
$$

for all $p(x)$ in $P$. The forward difference functional is the delta functional $e^{y t}-1$ and

$$
\left\langle e^{y t}-1 \mid p(x)\right\rangle=p(y)-p(0)
$$

The Abel functional is the delta functional $t e^{y t}$. We have

$$
\left\langle t e^{y t} \mid p(x)\right\rangle=p^{\prime}(y) .
$$

The Sheffer polynomials are defined by means of the following generating function

$$
\sum_{k=0}^{\infty} \frac{s_{k}(x)}{k !} t^{k}=\frac{1}{g(t)} e^{x t}
$$

Roman [34] proved the following theorem which is represented by the Sheffer polynomials (or Sheffer sequences) explicitly.

Theorem 2.1 Let $f(t)$ be a delta series and let $g(t)$ be an invertible series. Then there exists a unique sequence $s_{n}(x)$ of polynomials satisfying the orthogonality conditions

$$
\left\langle g(t) f(t)^{k} \mid s_{n}(x)\right\rangle=n ! \delta_{n, k}
$$

for all $k \in \mathbb{N}_{0}$.

The sequence $s_{n}(x)$ in (2.7) is the Sheffer polynomials for pair $(g(t), f(t))$, where $g(t)$ must be invertible and $f(t)$ must be delta series. The Sheffer polynomials for pair $(g(t), t)$ is the Appell polynomials or the Appell sequences for $g(t)$.

The Appell polynomials, the Bernoulli polynomials, the Euler polynomials, the Genocchi polynomials and the Genocchi polynomials of higher order belong to the family of the Sheffer polynomials (cf. [31, 34-36]). 
The Sheffer polynomials satisfy the following relations:

$$
s_{n}(x)=g(t)^{-1} x^{n},
$$

derivative formula

$$
t s_{n}(x)=s_{n}^{\prime}(x)=n s_{n-1}(x)
$$

recurrence formula

$$
s_{n+1}(x)=\left(x-\frac{g^{\prime}(t)}{g(t)}\right) s_{n}(x)
$$

expansion theorem

$$
h(t)=\sum_{k=0}^{\infty} \frac{\left\langle h(t) \mid s_{k}(x)\right\rangle}{k !} g(t) t^{k}
$$

multiplication theorem, for $\alpha \neq 0$,

$$
s_{n}(\alpha x)=\alpha^{n} \frac{g(t)}{g\left(\frac{t}{\alpha}\right)} s_{n}(x)
$$

and

$$
\langle h(t) \mid p(a x)\rangle=\langle h(a t) \mid p(x)\rangle .
$$

\section{The Apostol-type polynomials on $\mathcal{F}$}

We see from Definition 1.6 and (2.6) that the generalized Apostol-type polynomials $\mathcal{F}_{n}^{(\alpha)}(x ; \lambda ; \mu ; v)$ also belong to the Sheffer polynomials where $g(t)=\left(\frac{\lambda e^{t}+1}{2^{\mu} t^{v}}\right)^{\alpha}$.

In this section, by using the properties of the Sheffer sequences and also the Appell sequences, we prove many fundamental properties of the generalized Apostol-type polynomials $\mathcal{F}_{n}^{(\alpha)}(x ; \lambda ; \mu ; \nu)$ defined by $(1.16)$.

By using (2.8) and (1.16), we arrive at the following lemma.

\section{Lemma 3.1}

$$
\mathcal{F}_{n}^{(\alpha)}(x ; \lambda ; \mu ; \nu)=\left(\frac{2^{\mu} t^{\nu}}{\lambda e^{t}+1}\right)^{\alpha} x^{n}
$$

\section{Theorem 3.2}

$$
\left\langle\frac{\left(\lambda e^{t}+1\right)^{k}}{t^{\nu-1}} \mid \mathcal{F}_{n}^{(1)}(x ; \lambda ; \mu ; \nu)\right\rangle=2^{\mu} \lambda^{k-1} n(k-1) ! \sum_{j=0}^{k-1}\left(1+\frac{1}{\lambda}\right)^{k-j-1} \frac{S(n-1, j)}{(k-j-1) !},
$$

where $\mathcal{F}_{n}^{(1)}(x ; \lambda ; \mu ; v)$ and $S(a, b)$ denote the first-order generalized Apostol-type polynomials and the Stirling numbers of the second kind, respectively. 
Proof By Lemma 3.1, we obtain

$$
\left\langle\frac{\left(\lambda e^{t}+1\right)^{k}}{t^{\nu-1}} \mid \mathcal{F}_{n}^{(1)}(x ; \lambda ; \mu ; \nu)\right\rangle=\left\langle\frac{\left(\lambda e^{t}+1\right)^{k}}{t^{\nu-1}} \mid \frac{2^{\mu} t^{\nu}}{\lambda e^{t}+1} x^{n}\right\rangle .
$$

By using (2.3) and (2.9), we get

$$
\begin{aligned}
& \left\langle\frac{\left(\lambda e^{t}+1\right)^{k}}{t^{\nu-1}} \mid \mathcal{F}_{n}^{(1)}(x ; \lambda ; \mu ; \nu)\right\rangle \\
& \quad=2^{\mu} \lambda^{k-1} n \sum_{j=0}^{k-1} \frac{(k-1) !}{(k-j-1) !}\left(1+\frac{1}{\lambda}\right)^{k-j-1}\left\langle\frac{\left(e^{t}-1\right)^{j}}{j !} \mid x^{n-1}\right\rangle .
\end{aligned}
$$

Setting

$$
S(n-1, j)=\frac{1}{j !}\left\langle\left(e^{t}-1\right)^{j} \mid x^{n-1}\right\rangle
$$

where $S(n-1, j)$ denotes the Stirling numbers of second kind ( $c f .[34$, p.59]) in (3.3), we arrive at the desired result.

We deduce the following formulas.

Letting $\lambda \longmapsto-\lambda$, taking $\mu=0$ and $v=1$ in (3.2) and noting relation (1.17), we deduce the following result.

Corollary 3.3 (see [32, Remark 19])

$$
\left\langle\left(1-\lambda e^{t}\right)^{k} \mid \mathcal{B}_{n}(x ; \lambda)\right\rangle=(-1)^{k} \lambda^{k-1} n(k-1) ! \sum_{j=0}^{k-1}\left(1-\frac{1}{\lambda}\right)^{k-j-1} \frac{S(n-1, j)}{(k-j-1) !},
$$

where $\mathcal{B}_{n}(x ; \lambda)$ and $S(a, b)$ denote the Apostol-Bernoulli polynomials and the Stirling numbers of the second kind, respectively.

Taking $\mu=1$ and $v=0$ in (3.2) and noting relation (1.18), we deduce the following result.

Corollary 3.4 (see [32, Remark 21])

$$
\left\langle t\left(\lambda e^{t}+1\right)^{k} \mid \mathcal{E}_{n}(x ; \lambda)\right\rangle=2 \lambda^{k-1} n(k-1) ! \sum_{j=0}^{k-1}\left(1+\frac{1}{\lambda}\right)^{k-j-1} \frac{S(n-1, j)}{(k-j-1) !},
$$

where $\mathcal{E}_{n}(x ; \lambda)$ and $S(a, b)$ denote the Apostol-Euler polynomials and the Stirling numbers of the second kind, respectively.

Taking $\mu=v=1$ in (3.2) and noting relation (1.19), we deduce the following result.

Corollary 3.5 (see [32, Remark 20])

$$
\left\langle\left(\lambda e^{t}+1\right)^{k} \mid \mathcal{G}_{n}(x ; \lambda)\right\rangle=2 \lambda^{k-1} n(k-1) ! \sum_{j=0}^{k-1}\left(1+\frac{1}{\lambda}\right)^{k-j-1} \frac{S(n-1, j)}{(k-j-1) !},
$$


where $\mathcal{G}_{n}(x ; \lambda)$ and $S(a, b)$ denote the Apostol-Genocchi polynomials and the Stirling numbers of the second kind, respectively.

Setting $\lambda=1$ in (3.6), we deduce Theorem 2 in the work [31, p.758, Theorem 2].

\section{Corollary 3.6}

$$
\left\langle\left(e^{t}+1\right)^{k} \mid G_{n}(x)\right\rangle=2 n(k-1) ! \sum_{j=0}^{k-1} 2^{k-j-1} \frac{S(n-1, j)}{(k-j-1) !},
$$

where $G_{n}(x)$ and $S(a, b)$ denote the Genocchi polynomials and the Stirling numbers of the second kind, respectively.

Letting $k \mapsto m$, taking $\lambda=-\left(\frac{\beta}{a}\right)^{b}, \mu=1-\kappa, \nu=\kappa$ in (3.2) and noting relation (1.20), thus we deduce the following formulas of the polynomials $\mathcal{Y}_{n, \beta}(x ; \kappa, a, b)$.

\section{Corollary 3.7}

$$
\begin{aligned}
& \left\langle\left[1-\left(\frac{\beta}{a}\right)^{b} e^{t}\right]^{m} t^{1-\kappa} \mid \mathcal{Y}_{n, \beta}(x ; \kappa, a, b)\right\rangle \\
& \quad=(-1)^{m} 2^{1-\kappa} \beta^{b(m-1)} a^{-b m} n(m-1) ! \sum_{j=0}^{m-1}\left[1-\left(\frac{a}{\beta}\right)^{b}\right]^{m-j-1} \frac{S(n-1, j)}{(m-j-1) !},
\end{aligned}
$$

where $\mathcal{Y}_{n, \beta}(x ; \kappa, a, b)$ and $S(a, b)$ denote the generalization of Apostol type polynomials defined by (1.14) and the Stirling numbers of the second kind, respectively.

By using (2.9), we arrive at the following lemma.

\section{Lemma 3.8}

$$
t \mathcal{F}_{n}^{(\alpha)}(x ; \lambda ; \mu ; v)=n \mathcal{F}_{n-1}^{(\alpha)}(x ; \lambda ; \mu ; v)
$$

Remark 3.9 An alternative proof of Lemma 3.8 is also obtained from (1.16) by using derivative with respect to $x$. By Lemma 3.8, one can see that

$$
\frac{1}{t} \mathcal{F}_{n}^{(\alpha)}(x ; \lambda ; \mu ; \nu)=\frac{1}{n+1} \mathcal{F}_{n+1}^{(\alpha)}(x ; \lambda ; \mu ; \nu) .
$$

\section{Theorem 3.10}

$$
\left(\frac{t^{\nu-1}}{\lambda e^{t}+1}\right) \mathcal{F}_{n}^{(\alpha)}(x ; \lambda ; \mu ; \nu)=\frac{1}{2^{\mu}(n+1)} \mathcal{F}_{n+1}^{(\alpha+1)}(x ; \lambda ; \mu ; \nu) .
$$

Proof By Lemma 3.1, we obtain

$$
\left(\frac{t^{\nu-1}}{\lambda e^{t}+1}\right) \mathcal{F}_{n}^{(\alpha)}(x ; \lambda ; \mu ; \nu)=\frac{t^{\nu-1}}{\lambda e^{t}+1}\left(\frac{2^{\mu} t^{\nu}}{\lambda e^{t}+1}\right)^{\alpha} x^{n}
$$


After some calculations in the above equation, we have

$$
\left(\frac{t^{\nu-1}}{\lambda e^{t}+1}\right) \mathcal{F}_{n}^{(\alpha)}(x ; \lambda ; \mu ; v)=\frac{1}{2^{\mu} t}\left(\frac{2^{\mu} t^{\nu}}{\lambda e^{t}+1}\right)^{\alpha+1} x^{n}
$$

Using (1.16) and (3.10), we obtain the desired result.

Letting $\lambda \longmapsto-\lambda$, taking $\mu=0$ and $v=1$ in (3.11) and noting relation (1.17), we deduce the following result.

Corollary 3.11 (see [32, Remark 32])

$$
\left(\frac{1}{1-\lambda e^{t}}\right) \mathcal{B}_{n}^{(\alpha)}(x ; \lambda)=\frac{1}{n+1} \mathcal{B}_{n+1}^{(\alpha+1)}(x ; \lambda) .
$$

Taking $\mu=1$ and $\nu=0$ in (3.11) and noting relation (1.18), we deduce the following result.

Corollary 3.12 (see [32, Remark 33])

$$
\frac{1}{t\left(\lambda e^{t}+1\right)} \mathcal{E}_{n}^{(\alpha)}(x ; \lambda)=\frac{1}{2(n+1)} \mathcal{E}_{n+1}^{(\alpha+1)}(x ; \lambda) .
$$

Taking $\mu=v=1$ in (3.11) and noting relation (1.19), we deduce the following result.

Corollary 3.13 (see [32, Remark 34])

$$
\left(\frac{1}{\lambda e^{t}+1}\right) \mathcal{G}_{n}^{(\alpha)}(x ; \lambda)=\frac{1}{2(n+1)} \mathcal{G}_{n+1}^{(\alpha+1)}(x ; \lambda)
$$

Setting $\lambda=1$ in the above equation, we deduce Lemma 3 in [31, p.758].

\section{Corollary 3.14}

$$
\left(\frac{1}{e^{t}+1}\right) G_{n}^{(\alpha)}(x)=\frac{1}{2(n+1)} G_{n+1}^{(\alpha+1)}(x) .
$$

Taking $\lambda=-\left(\frac{\beta}{a}\right)^{b}, \mu=1-\kappa, \nu=\kappa$ in (3.11) and noting relation (1.21), we deduce

\section{Corollary 3.15}

$$
\frac{-t^{\kappa-1}}{a^{b}\left[1-\left(\frac{\beta}{a}\right)^{b} e^{t}\right]} \mathcal{Y}_{n, \beta}^{(\alpha)}(x ; \kappa, a, b)=\frac{1}{2^{1-\kappa}(n+1)} \mathcal{Y}_{n+1, \beta}^{(\alpha+1)}(x ; \kappa, a, b) .
$$

An integral representation of $\left\langle\frac{e^{t a}-1}{2 t} \mid \mathcal{F}_{n}^{(\alpha)}(x ; \lambda ; \mu ; \nu)\right\rangle$ is given by the following theorem.

\section{Theorem 3.16}

$$
\left\langle\frac{e^{t a}-1}{2 t} \mid \mathcal{F}_{n}^{(\alpha)}(x ; \lambda ; \mu ; v)\right\rangle=\frac{1}{2} \int_{0}^{a} \mathcal{F}_{n}^{(\alpha)}(x ; \lambda ; \mu ; \nu) d x .
$$


Proof By using Lemma 3.8, we have

$$
\left\langle\frac{e^{t a}-1}{2 t} \mid \mathcal{F}_{n}^{(\alpha)}(x ; \lambda ; \mu ; \nu)\right\rangle=\left\langle\frac{e^{t a}-1}{2 t} \mid \frac{1}{n+1} t \mathcal{F}_{n+1}^{(\alpha)}(x ; \lambda ; \mu ; \nu)\right\rangle .
$$

By (2.3), we obtain

$$
\left\langle\frac{e^{t a}-1}{2 t} \mid \mathcal{F}_{n}^{(\alpha)}(x ; \lambda ; \mu ; \nu)\right\rangle=\frac{1}{2(n+1)}\left\langle e^{t a}-1 \mid \mathcal{F}_{n+1}^{(\alpha)}(x ; \lambda ; \mu ; \nu)\right\rangle .
$$

Using (2.5), we obtain the desired result.

Setting $\lambda=\mu=v=1$ in (3.19) and noting relation (1.19), we deduce the Theorem 3 in [31, p.758].

\section{Corollary 3.17}

$$
\left\langle\frac{e^{t a}-1}{2 t} \mid G_{n}^{(\alpha)}(x)\right\rangle=\frac{1}{2} \int_{0}^{a} G_{n}^{(\alpha)}(x) d x .
$$

A recurrence formula for $\mathcal{F}_{n}^{(\alpha)}(x ; \lambda ; \mu ; \nu)$ is given by the next theorem.

Theorem 3.18 (Recurrence formula)

$$
\begin{aligned}
& \mathcal{F}_{n+v}^{(\alpha+1)}(x ; \lambda ; \mu ; \nu) \\
& \quad=\frac{2^{\mu}(n+1)(n+1) !}{\alpha(n+v) !}\left[\left(1-\frac{\alpha v}{n+1}\right) \mathcal{F}_{n+1}^{(\alpha)}(x ; \lambda ; \mu ; v)+(\alpha-x) \mathcal{F}_{n}^{(\alpha)}(x ; \lambda ; \mu ; \nu)\right] .
\end{aligned}
$$

Proof Setting

$$
g(t)=\left(\frac{\lambda e^{t}+1}{2^{\mu} t^{\nu}}\right)^{\alpha}
$$

in (2.10), one can obtain

$$
\begin{aligned}
& \mathcal{F}_{n+1}^{(\alpha)}(x ; \lambda ; \mu ; \nu) \\
& \quad=\left(x-\alpha+\frac{\alpha}{\lambda e^{t}+1}+\frac{\alpha \nu}{t}\right) \mathcal{F}_{n}^{(\alpha)}(x ; \lambda ; \mu ; \nu) \\
& \quad=(x-\alpha) \mathcal{F}_{n}^{(\alpha)}(x ; \lambda ; \mu ; \nu)+\frac{\alpha}{t^{\nu-1}} \cdot \frac{t^{\nu-1}}{\lambda e^{t}+1} \mathcal{F}_{n}^{(\alpha)}(x ; \lambda ; \mu ; \nu)+\alpha \nu \cdot \frac{1}{t} \mathcal{F}_{n}^{(\alpha)}(x ; \lambda ; \mu ; \nu) .
\end{aligned}
$$

By using Theorem 3.10 and (3.10), we have

$$
\begin{aligned}
\mathcal{F}_{n+1}^{(\alpha)}(x ; \lambda ; \mu ; \nu)= & (x-\alpha) \mathcal{F}_{n}^{(\alpha)}(x ; \lambda ; \mu ; \nu)+\frac{\alpha(n+\nu) !}{2^{\mu}(n+1)(n+1) !} \mathcal{F}_{n+v}^{(\alpha+1)}(x ; \lambda ; \mu ; \nu) \\
& +\frac{\alpha \nu}{n+1} \mathcal{F}_{n+1}^{(\alpha)}(x ; \lambda ; \mu ; \nu) .
\end{aligned}
$$

After some calculations in the above equation, we get the desired result. 
Letting $\lambda \longmapsto-\lambda$, taking $\mu=0$ and $v=1$ in (3.21) and noting relation (1.17), we deduce the following known result.

Corollary 3.19 (see, e.g., [32, Remark 38])

$$
\mathcal{B}_{n+1}^{(\alpha+1)}(x ; \lambda)=\frac{1}{\alpha}\left[(\alpha-n-1) \mathcal{B}_{n+1}^{(\alpha)}(x ; \lambda)+(n+1)(x-\alpha) \mathcal{B}_{n}^{(\alpha)}(x ; \lambda)\right] .
$$

Taking $\mu=1$ and $v=0$ in (3.21) and noting relation (1.18), we deduce the following known result.

Corollary 3.20 (see, e.g., [32, Remark 39])

$$
\mathcal{E}_{n}^{(\alpha+1)}(x ; \lambda)=\frac{2(n+1)^{2}}{\alpha}\left[\mathcal{E}_{n+1}^{(\alpha)}(x ; \lambda)+(\alpha-x) \mathcal{E}_{n}^{(\alpha)}(x ; \lambda)\right]
$$

Taking $\mu=v=1$ in (3.21) and noting relation (1.19), we deduce the following known result.

Corollary 3.21 (see, e.g., [32, Remark 40])

$$
\mathcal{G}_{n+1}^{(\alpha+1)}(x ; \lambda)=\frac{2}{\alpha}\left[(n-\alpha+1) \mathcal{G}_{n+1}^{(\alpha)}(x ; \lambda)+(n+1)(\alpha-x) \mathcal{G}_{n}^{(\alpha)}(x ; \lambda)\right] .
$$

Setting $\lambda=1$ in the above equation, we have the following.

Corollary 3.22 (see [31, p.759, Theorem 4])

$$
G_{n+1}^{(\alpha+1)}(x)=\frac{2}{\alpha}\left[(n-\alpha+1) G_{n+1}^{(\alpha)}(x)+(n+1)(\alpha-x) G_{n}^{(\alpha)}(x)\right] .
$$

Taking $\lambda=-\left(\frac{\beta}{a}\right)^{b}, \mu=1-\kappa, \nu=\kappa$ in (3.21) and noting relation (1.21), thus we deduce the following result.

\section{Corollary 3.23}

$$
\begin{aligned}
\mathcal{Y}_{n+\kappa, \beta}^{(\alpha+1)}(x ; \kappa, a, b)= & \frac{2^{1-\kappa}(n+1)(n+1) !}{\alpha a^{b}(n+\kappa) !} \\
& \times\left[\left(\frac{\alpha \kappa}{n+1}-1\right) \mathcal{Y}_{n+1, \beta}^{(\alpha)}(x ; k, a, b)+(x-\alpha) \mathcal{Y}_{n, \beta}^{(\alpha)}(x ; \kappa, a, b)\right] .
\end{aligned}
$$

Competing interests

The authors declare that they have no competing interests.

\section{Authors' contributions}

All authors contributed equally in writing this paper, and read and approved the final manuscript.

\section{Author details}

'Department of Mathematics, Yangzhou University, Yangzhou, Jiangsu 225002, People's Republic of China. ${ }^{2}$ Department of Mathematics, Chongqing Normal University, Chongqing Higher Education Mega Center, Huxi Campus, Chongqing, 401331, People's Republic of China. 


\section{Acknowledgements}

Dedicated to Professor Hari M. Srivastava on the occasion of his seventy-third birth anniversary. The authors sincerely thank the referees for their valuable suggestions and comments. The present investigation was supported by the National Natural Science Foundation of China under Grant 11226281, Fund of Science and Innovation of Yangzhou University, China under Grant 2012CXJ005, Natural Science Foundation Project of Chongging, China under Grant CSTC2011 JJA00024, Research Project of Science and Technology of Chongaing Education Commission, China under Grant KJ120625, Fund of Chongaing Normal University, China under Grant 10XLR017 and 2011XLZ07 and Program of Chongqing Innovation Team Project in University under Grant No. KJTD201308.

\section{Received: 10 December 2012 Accepted: 20 June 2013 Published: 5 July 2013}

\section{References}

1. Sándor, J, Crstici, B: Handbook of Number Theory, vol. II. Kluwer Academic, Dordrecht (2004)

2. Srivastava, HM, Choi, J: Series Associated with the Zeta and Related Functions. Kluwer Academic, Dordrecht (2001)

3. Apostol, TM: On the Lerch zeta function. Pac. J. Math. 1, 161-167 (1951)

4. Srivastava, HM: Some formulas for the Bernoulli and Euler polynomials at rational arguments. Math. Proc. Camb. Philos. Soc. 129, 77-84 (2000)

5. Luo, Q-M, Srivastava, HM: Some generalizations of the Apostol-Bernoulli and Apostol-Euler polynomials. J. Math. Anal. Appl. 308, 290-320 (2005)

6. Luo, Q-M: Apostol-Euler polynomials of higher order and Gaussian hypergeometric functions. Taiwan. J. Math. 10 917-925 (2006)

7. Horadam, AF: Genocchi polynomials. In: Proceedings of the Fourth International Conference on Fibonacci Numbers and Their Applications, pp. 145-166. Kluwer Academic, Dordrecht (1991)

8. Horadam, AF: Negative order Genocchi polynomials. Fibonacci Q. 30, 21-34 (1992)

9. Horadam, AF: Generation of Genocchi polynomials of first order by recurrence relations. Fibonacci Q. 30, 239-243 (1992)

10. Jang, L-C, Kim, T: On the distribution of the $q$-Euler polynomials and the $q$-Genocchi polynomials of higher order. J. Inequal. Appl. 2008, 1-9 (2008)

11. Kim, T, Jang, L-C, Pak, HK: A note on q-Euler numbers and Genocchi numbers. Proc. Jpn. Acad., Ser. A, Math. Sci. 77, 139-141 (2001)

12. Luo, Q-M: Extension for the Genocchi polynomials and its Fourier expansions and integral representations. Osaka J. Math. 48, 291-309 (2011)

13. Ozden, H, Simsek, Y, Srivastava, HM: A unified presentation of the generating functions of the generalized Bernoulli, Euler and Genocchi polynomials. Comput. Math. Appl. 60, 2779-2787 (2010)

14. Ozden, $\mathrm{H}$ : Generating functions of the unified representation of the Bernoulli, Euler and Genocchi polynomials of higher order. Proceedings of the International Conference on Numerical Analysis and Applied Mathematics 2011. AIP Conf. Proc. 1389, 349-352 (2011)

15. Özarslan, MA: Unified Apostol-Bernoullli, Euler and Genocchi polynomials. Comput. Math. Appl. 62, 2452-2462 (2011)

16. Luo, Q-M, Srivastava, HM: Some generalizations of the Apostol-Genocchi polynomials and the Stirling numbers of the second kind. Appl. Math. Comput. 217, 5702-5728 (2011)

17. Luo, Q-M, Srivastava, HM: Some relationships between the Apostol-Bernoulli and Apostol-Euler polynomials. Comput. Math. Appl. 51, 631-642 (2006)

18. Luo, Q-M: The multiplication formulas for the Apostol-Bernoulli and Apostol-Euler polynomials of higher order. Integral Transforms Spec. Funct. 20, 377-391 (2009)

19. Lu, D-Q, Srivastava, HM: Some series identities involving the generalized Apostol-type and related polynomials. Comput. Math. Appl. 62, 3591-3602 (2011)

20. Srivastava, HM: Some generalizations and basic (or $q^{-}$) extensions of the Bernoulli, Euler and Genocchi polynomials. Appl. Math. Inform. Sci. 5, 390-444 (2011)

21. Srivastava, HM, Kurt, B, Simsek, Y: Some families of Genocchi type polynomials and their interpolation functions. Integral Transforms Spec. Funct. 23, 919-938 (2012); see also Corrigendum. Integral Transforms Spec. Funct. 23, 939-940 (2012)

22. Srivastava, HM, Choi, J: Zeta and q-Zeta Functions and Associated Series and Integrals. Elsevier, Amsterdam (2012)

23. Srivastava, HM, Özarslan, MA, Kaanuglu, C: Some generalized Lagrange-based Apostol-Bernoulli, Apostol-Euler and Apostol-Genocchi polynomials. Russ. J. Math. Phys. 20, 110-120 (2013)

24. Luo, Q-M: Fourier expansions and integral representations for the Apostol-Bernoulli and Apostol-Euler polynomials. Math. Comput. 78, 2193-2208 (2009)

25. Luo, Q-M: An explicit formula for the Euler polynomials of higher order. Appl. Math. Inform. Sci. 3(1), 53-58 (2009)

26. Luo, Q-M: Some formulas for Apostol-Euler polynomials associated with Hurwitz zeta function at rational arguments. Appl. Anal. Discrete Math. 3, 336-346 (2009)

27. Luo, Q-M: Some results for the q-Bernoulli and q-Euler polynomials. J. Math. Anal. Appl. 363, 7-18 (2010)

28. Luo, Q-M: An explicit relationship between the generalized Apostol-Bernoulli and Apostol-Euler polynomials associated with $\lambda$-Stirling numbers of the second kind. Houst. J. Math. 36, 1159-1171 (2010)

29. Luo, Q-M, Srivastava, HM: q-Extensions of some relationships between the Bernoulli and Euler polynomials. Taiwan. J. Math. 15, 241-257 (2011)

30. Luo, Q-M, Zhou, Y: Extension of the Genocchi polynomials and its q-analogue. Util. Math. 85, 281-297 (2011)

31. Dere, R, Simsek, Y: Genocchi polynomials associated with the Umbral algebra. Appl. Math. Comput. 218, 756-761 (2011)

32. Dere, R, Simsek, Y: Unification of the three families of generalized Apostol type polynomials on the Umbral algebra. arXiv:1110.2047v1

33. Dere, R, Simsek, Y, Srivastava, HM: A unified presentation of three families of generalized Apostol-type polynomials based upon the theory of the umbral calculus and the umbral algebra. J. Number Theory 133, 3245-3263 (2013)

34. Roman, S: The Umbral Calculus. Dover, New York (2005) 
35. Blasiak, P, Dattoli, G, Horzela, A, Penson, KA: Representations of monomiality principle with Sheffer-type polynomials and boson normal ordering. Phys. Lett. A 352, 7-12 (2006)

36. Dattoli, G, Migliorati, M, Srivastava, HM: Sheffer polynomials, monomiality principle, algebraic methods and the theory of classical polynomials. Math. Comput. Model. 45, 1033-1041 (2007)

doi:10.1186/1687-1847-2013-201

Cite this article as: Lu et al.: Some results for Apostol-type polynomials associated with umbral algebra. Advances in Difference Equations 2013 2013:201.

Submit your manuscript to a SpringerOpen ${ }^{\odot}$ journal and benefit from:

- Convenient online submission

- Rigorous peer review

- Immediate publication on acceptance

- Open access: articles freely available online

- High visibility within the field

- Retaining the copyright to your article

Submit your next manuscript at $>$ springeropen.com 